\title{
Transatlantica
}

Revue d'études américaines. American Studies Journal

\section{Laura Jensen. Patriots, Settlers and the Origins of} American Social Policy.

Cambridge : Cambridge University Press, 2003. 244p.

\section{Elisabeth Boulot et Evelyne Thévenard}

\section{CpenEdition}

\section{Journals}

Édition électronique

URL : http://journals.openedition.org/transatlantica/820

DOI : $10.4000 /$ transatlantica.820

ISSN : 1765-2766

Éditeur

AFEA

\section{Référence électronique}

Elisabeth Boulot et Evelyne Thévenard, «Laura Jensen. Patriots, Settlers and the Origins of American Social Policy. », Transatlantica [En ligne], 1 | 2005, mis en ligne le 22 avril 2006, consulté le 29 avril 2021. URL : http://journals.openedition.org/transatlantica/820 ; DOI : https://doi.org/10.4000/transatlantica. 820

Ce document a été généré automatiquement le 29 avril 2021.

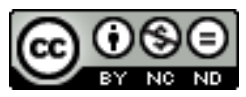

Transatlantica - Revue d'études américaines est mis à disposition selon les termes de la licence Creative Commons Attribution - Pas d'Utilisation Commerciale - Pas de Modification 4.0 International. 


\section{Laura Jensen. Patriots, Settlers and the Origins of American Social Policy.}

Cambridge : Cambridge University Press, 2003. 244p.

Elisabeth Boulot et Evelyne Thévenard

Dans son ouvrage sur les origines de l'Etat-Providence aux Etats-Unis, Laura Jensen réfute la thèse de l'historiographie classique, qui privilégie le New Deal et la Great Society dans le processus d'élaboration de la protection sociale américaine. Mais surtout, elle s'applique à démontrer que, contrairement à la thèse de Theda Skocpol (Protecting Soldiers and Mothers), les droits sociaux et leur contribution à la construction de la nation et à la définition de la citoyenneté ne datent pas des pensions de la Guerre de Sécession. Les pensions accordées - après de multiples débats - par le Congrès, aux combattants de la Guerre d'Indépendance, et les terres allouées par l'Etat fédéral à diverses catégories de citoyens entre 1789 et 1862 ont eu un impact déterminant sur la constitution d'une armée, la mise en place de structures administratives, l'expansion vers l'Ouest, l'élimination des Indiens et ont jeté les bases du type d'Etat-Providence dont devaient se doter les Etats-Unis à partir du début du 20e siècle. L'attribution de droits à certaines catégories, selon des critères spécifiques (entitlements) et non sur la base de la citoyenneté, a été l'instrument d'une politique délibérée de l'Etat américain depuis la naissance de la nation.

Afin de bien marquer la pertinence de cette analyse historique, à un moment où des pans entiers de la protection sociale américaine sont remis en question, l'auteur nous invite d'abord à une réflexion sur le terme entitlement (droits sociaux, droits acquis), dont la définition ne fait pas l'objet d'un consensus parmi les experts, mais qui est incontestablement porteur d'une charge politique et idéologique. Si, jusqu'aux années 90 le terme désignait en général le système d'assurance sociale (pensions et assurance maladie des retraités), basé sur les cotisations, par opposition à l'aide sociale, il semblerait qu'à l'heure actuelle la distinction soit moins marquée et que par ailleurs le mot entitlement prenne, dans le discours conservateur, des connotations négatives liées au coût de ces dispositifs, et à l'hypothèque qu'ils représentent pour l'avenir des générations futures. Ces droits sociaux, malgré les efforts de certains juristes 
progressistes pendant les années 60 ne sont pas inscrits dans la Constitution et sont soumis à des critères d'accès définis par le Congrès. Laura Jensen va montrer que la politique d'attribution des pensions pour les vétérans comme celle de l'attribution de terres aux pionniers dans le courant $\mathrm{du} 19^{\mathrm{e}}$ siècle a très tôt posé le problème des critères d'accès à ces droits, créant des catégories d'inclusion et d'exclusion, des groupes de références d'où certains étaient exclus - et ceci reste la caractéristique de l'Etat-Providence américain.

Dans le premier chapitre, l'auteur expose la thèse qu'elle défend et la problématique de l'ouvrage : l'étude du comment, du pourquoi mais aussi des effets de ces choix en matière d'aide sociale, effectués à l'échelon national dès les origines de la nation américaine.

2 Les chapitres 2 et 3 sont consacrés à une étude minutieuse et très documentée des différentes dispositions votées par le Congrès sur la question des pensions accordées à certaines catégories d'anciens combattants des deux guerres d'indépendance et analyse les raisons qui ont conduit ce dernier à agir de la sorte. Elles sont à la fois constitutionnelles, conjoncturelles et institutionnelles.

3 En ce qui concerne les patriots (combattants de la Guerre d'Indépendance), si le versement de pensions d'invalidité, voté par le Congrès Continental dès le début des hostilités ne fut jamais contesté (le dispositif fut renouvelé et élargi à plusieurs reprises jusqu'en 1828), l'attribution de pensions de retraite fut très controversée, dans son principe et son application. Les Articles de Confédération comme la Constitution de 1787 prévoient que le Congrès puisse lever et entretenir des armées seulement en temps de guerre et pourvoir à l'organisation, l'armement et à la discipline de la milice employée au service des Etats-Unis mais n'instaure pas de système de conscription. La nomination des officiers relève des Etats. Pour les patriotes, dès le début de la révolution américaine seule la milice (une armée libre constitués d'hommes libres) par opposition à une armée de métier, pouvait sacrifier son intérêt personnel pour la défense du bien commun. La crainte de voir se constituer une armée permanente, avec la menace qu'elle représentait pour les libertés céda à la nécessité de créer un corps d'officiers, les citoyens-soldats s'avérant incapables à eux seuls de gagner la guerre contre l'Angleterre. En 1778, la décision du Congrès Continental, après des débats longs et houleux, d'attribuer une pension pendant 7 ans aux officiers ainsi qu'une allocation forfaitaire de 80 dollars aux autres combattants marqua le début de plusieurs décennies de controverses sur les critères d'accès à ces avantages sociaux.

4 L'attribution de ces pensions versées à titre individuel et souvent à un nombre d'ayant droits plus élevés que prévu nécessita des prélèvements importants sur les recettes fiscales. Ceci conduisit le Sénat en 1818 à faire ces choix certes discriminatoires mais rendus nécessaires afin de préserver l'équilibre financier de la jeune nation tout en assurant son développement.

5 Enfin les institutions américaines qui reposent sur le principe d'un système de gouvernement décentralisé et sur celui des échelles de gouvernance conduisirent le gouvernement fédéral à vouloir asseoir son autorité face à des Etats souverains, jaloux de leurs prérogatives, afin d'atteindre ses objectifs : la défense et l'indépendance du pays, puis le contrôle de son expansion territoriale.

6 Le Pension Act de 1818, qui accordait une pension à tous les citoyens américains âgés, ayant servi au moins 9 mois dans l'armée continentale, et qui se trouvaient dans le besoin est, selon l'auteur, l'acte fondateur de la politique sociale américaine, ciblée sur des 
groupes particuliers. Les débats au Congrès entre 1818 et 1832 sur l'extension des pensions à tous les vétérans nécessiteux se déroula sur fond de querelle à propos des tarifs douaniers, le Sud accusant le Nord d'imposer ces taxes nuisibles à son économie afin de verser de généreuses pensions aux vétérans plus nombreux dans les Etats du Nord. Les mêmes discours dénonçaient l'injustice des pensions, la menace pour les valeurs américaines de travail et de moralité et l'extension du pouvoir fédéral au dépens des Etats.

7 Malgré les abus, et l'explosion du nombre des ayant droits, le Congrès, en dette de gratitude envers les fondateurs de la Nation, et pour sceller l'unité nationale élargit une fois de plus, en 1836, le nombre des bénéficiaires, en accordant une pension à vie aux veuves et orphelins de combattants morts pendant la guerre, ou qui décédaient des suites de leurs blessures.

8 Ces pensions, et les débats qui ont préfiguré ceux qui eurent lieu après la Guerre de Sécession (entre autres la controverse sur les droits basés sur le mérite et ceux basés sur le besoin) ont eu un impact important sur la notion de citoyenneté et la perception des liens entre l'Etat et la société civile. En outre, elles ont permis l'établissement d'une armée essentielle à la construction de la nation, elles ont accéléré le développement d'un système administratif et fiscal centralisé, elles ont contribué à mettre en place le système de commissions au Congrès et à renforcer la légitimité du pouvoir judiciaire fédéral chargé de l'adjudication des recours.

9 Si l' attribution de terres (land grants) aux soldats de la Révolution (selon leur rang) procédait au départ des mêmes causes que l'attribution de pensions: attirer des combattants motivés et compétents, elle fut bientôt envisagée dans une perspective expansionniste, afin de créer des avant-postes destinés à protéger les nouveaux territoires du Sud et de l'Ouest des incursions indiennes et étrangères (chapitres 4 et 5). Mais les vétérans n'étaient pas les seuls à avoir des visées sur le domaine public. Malgré l'interdiction votée par le Congrès en 1785 de squatter les terres inoccupées, les occupations illégales de territoires se multipliaient. Bien qu'Hamilton ait recommandé, en 1790, de privilégier la vente des terres comme source de revenus pour l'Etat, le souci d'encourager le peuplement de l'Ouest l'emporta peu à peu sur les considérations financières. Après la victoire de 1812, quand la jeune république se tourna résolument vers l'Ouest, la question des modalités de la distribution des terres, bien que moins controversée que celle des pensions (c'était une politique distributrice et non redistributrice), fut cependant plus complexe pour le président et le Congrès car elle se trouva liée à d'autres questions épineuses, comme la politique étrangère, la politique commerciale, et l'esclavage. Après le déplacement forcé des Indiens à la suite du Indian Removal Act (1830), le Congrès devait à deux reprises, en 1830 et en 1841, assouplir les règles d'acquisition des terres et les sanctions contre les squatters. De même que les pensions avaient récompensé les patriotes, l'attribution de terres récompensaient les pionniers (settlers) et les fermiers-citoyens que les Démocrates comme les Whigs affirmaient vouloir défendre contre les spéculateurs.

Le vote par le Congrès, au début des années 1850, de plusieurs mesures accordant des terres à tous les anciens combattants de toutes les guerres depuis 1775, et la vente de ces terres aux spéculateurs déclencha l'indignation des partisans d'une réforme agraire que le Congrès s'obstinait à refuser. Mais ces derniers finirent par l'emporter, avec le Homestead Act de 1862 qui constitua la première mesure de politique sociale quasi universelle (le Southern Homestead Act de 1866 inclut les sudistes et les noirs 
initialement exclus - ce qui en fit la première législation nationale à interdire la discrimination raciale). Quant au Pension Act de 1862 qui accordait des pensions aux vétérans de la guerre de Sécession, ses caractéristiques sont bien connues. Beaucoup plus généreux que ses prédécesseurs, ses avantages furent étendus à un nombre croissant de bénéficiaires au fil des années. Là où Theda Skocpol voit avant tout les effets pervers du clientélisme électoral, Laura Jensen, qui précise que le Congrès, en 1872 vota une loi accordant une pension aux combattants de la guerre de 1812 voit la volonté de renforcer la cohésion nationale et de récompenser la fidélité à l'Etat (les sudistes furent toujours exclus du bénéfice de ces pensions).

11 L'Etat-Providence américain, fondé non pas sur une conception universaliste de la protection sociale mais sur la division de la société en catégories juridiques d'ayant droits ne remonte donc pas au New Deal, ni aux pensions de la Guerre de Sécession, mais a des racines beaucoup plus anciennes. L'ouvrage de Laura Jensen, dense et documenté apporte une contribution originale à l'histoire de la politique sociale américaine et projette un éclairage nouveau sur une période qui n'est pas traditionnellement perçue comme l'une des étapes majeures de cette histoire.

INDEX

Thèmes : Recensions

\section{AUTEURS}

ELISABETH BOULOT

Université de Marne-la-Vallée / Université Paris 4 